\title{
A Two-Fingered Robot Gripper with Variable Stiffness Flexure Hinges Based on Shape Morphing
}

\author{
Hareesh Godaba, Aqeel Sajad, Navin Patel, Kaspar Althoefer, and Ketao Zhang*
}

\begin{abstract}
This paper presents a novel approach for developing robotic grippers with variable stiffness hinges for dexterous grasps. This approach for the first time uses pneumatically actuated pouch actuators to fold and unfold morphable flaps of flexure hinges thus change stiffness of the hinge. By varying the air pressure in pouch actuators, the flexure hinge morphs into a beam with various open sections while the flaps bend, enabling stiffness variation of the flexure hinge. This design allows 3D printing of the flexure hinge using printable soft filaments. Utilizing the variable stiffness flexure hinges as the joints of robotic fingers, a light-weight and low-cost two-fingered tendon driven robotic gripper is developed. The stiffness variation caused due to the shape morphing of flexure hinges is studied by conducting static tests on fabricated hinges with different flap angles and on a flexure hinge with flaps that are bent by pouch actuators subjected to various pressures. Multiple grasp modes of the two-fingered gripper are demonstrated by grasping objects with various geometric shapes. The gripper is then integrated with a robot manipulator in a teleoperation setup for conducting a pick-and-place operation in a confined environment.
\end{abstract}

\section{INTRODUCTION}

Robots are generally known to be devices made from tough materials with the aim of undertaking complex tasks. Whilst they are usually admired for their robustness and ability to accurately repeat tasks and consistently provide identical results every time, the rigid-bodied robotic systems have limitations in applications where safety and adaptability during interactions are crucial. For instance, conventional robotic grippers find it challenging to grasp objects made from fragile materials without damaging them and are limited in their ability to interact with objects of irregular shapes. In contrast, soft robotic grippers have been proven to be capable of grasping a wide variety of objects [1], [2].

To develop robotic grippers, the concept of underactuation has been widely adopted as an effective approach for embedding high number of degrees of freedom (DOFs) without increasing the number of actuators that need to be controlled [3]. An underactuated robotic gripper not only reduces the complexity of actuation and control systems but also brings in inherent compliance and adaptability to interact with the objects and working environments. There have been extensive developments of underactuated grippers using conventional kinematic joints such as the underactuated

\footnotetext{
*This work was partially supported by research awards from the Engineering and Physical Sciences Research Council (EPSRC) projects, National Centre for Nuclear Robotics (NCNR) EP/R02572X/1, NCNR Flexifunds Grant and Queen Mary University of London Start-up fund.

Centre for Advanced Robotics (ARQ), Queen Mary University of London h.godabalqmul.ac.uk, navin.pateleqmul.ac.uk, k.althoefer@qmul.ac.uk, ketao.zhang@qmul.ac.uk
}

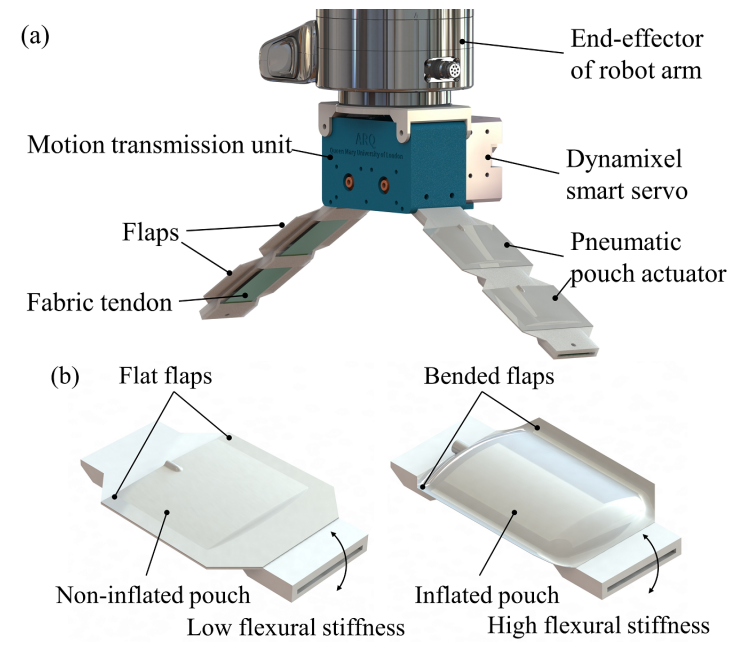

Fig. 1. (a) Schematic showing the proposed gripper with pneumatic pouches to vary the stiffness of the fingers, and (b) Variable stiffness flexure hinge with morphing flexible elements (flaps)

space robotic hands [4], [5], [6] developed at Universite Laval, the underactuated prosthetic hand [7] and the opensource 3D printed underactuated robotic gripper for research and education [8].

The aforementioned robotic grippers with conventional kinematic joints and rigid components lack inherent compliance, leading to limited ability to adapt themselves to different materials and shapes of objects. Inspired by living systems in nature, materials capable of large elastic deformation have been introduced in the development of soft robotic grippers [9] to bring in self-adaptability by leveraging the inherent compliance of soft/flexible materials. Using deformable materials, a wide range of underactuated robotic grippers with soft/flexible fingers have been developed in recent years. There are two main types of underactuated finger designs: one type uses flexure hinges replacing the conventional kinematic joints in the finger [10], [11], [8], [12], [13], [14], while the other type employs soft-bodied continuum robot fingers [15], [16]. Recently, there have been innovative developments of soft robotic grippers with integrated variable stiffness mechanisms [9], [17]. Further, tendon-driven robotic origami joints using thermally activated shape memory polymer to control the joint stiffness have been developed and integrated into a three-fingered origami gripper [18].

In this work, we propose a shape morphing inspired approach for developing 3D printable flexure hinges with 
adjustable stiffness to embed higher dexterity in a traditional tendon-driven underactuated gripping mechanism. A novel flexible robotic finger is developed by alternatively connecting the 3D printable flexure hinges with relatively stiffer segments and integrating pneumatically actuated pouches, which are used to control the morphing of flaps and, consequently, the stiffness of the flexure hinges. With a pair of flexible robotic fingers, a prototype of the two-fingered soft robotic gripper is developed and demonstrated in grasps of various objects (Fig. 1).

This work makes the following contributions:

1) A novel approach for designing and modelling a shape morphable flexure hinge with variable stiffness.

2) The integration of pneumatically actuated pouches with the flexure hinges for controlling the geometry and stiffness.

3) A light-weight and low-cost two-fingered soft gripper capable of multiple modes for grasping a wide range of objects and adapting to unstructured environments.

In the following sections, we first introduce the proposed design concept of variable stiffness flexure hinges with morphable flaps. Section III presents the design and fabrication of the flexible finger with variable stiffness hinges and the integration of pneumatic pouch actuator with the flexure hinges. Section IV details stiffness control of a single integrated flexure hinge by actively folding the flaps. Section $\mathrm{V}$ reports the control system and demonstrates the multiple modes for grasping a wide range of objects and adapting to unstructured environments, and the paper is then concluded in Section VI.

\section{VARIABle StifFNeSS FleXURE Hinge With FOLDABLE FLAPS}

Here we present the concept for designing variable stiffness flexure hinges based on the principles of load-resistance mechanisms of flat plates and beams with open cross sections. In terms of the beam theory, the moment of inertia (second moment of area) is used to describe the rigidity of a beam against flexure. Bending stiffness which is also known as flexural rigidity (defined as EI) varies along the length of the beam. This indicates that bending stiffness of a plate structure is proportional to the moment of inertia, which is a geometrical property of an area. According to the principles above, the flexural rigidity of a plate can be effectively controlled by changing its geometry, meaning the distribution of its points with regard to an axis of interest. As a result, this offers us novel avenues for designing stiffness controllable structures which can be used as variable stiffness joints for robot mechanisms. Inspired by origami-folding which transforms 2D flat templates into 3D, our proposed flexure hinge (Fig. 1(b)) consists of one plate beam and two flaps. The flaps can be folded around the edge of the plate thus resulting in a beam structure with various open sections thereby changing the moment of inertia and the resulting flexural rigidity of the overall structure. The mechanism of the change of the moment of inertia due to folding of flaps can be understood as follows.

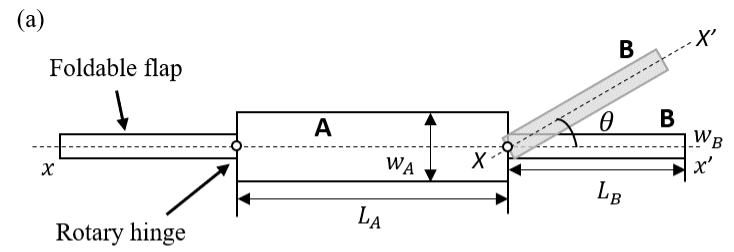

(b)

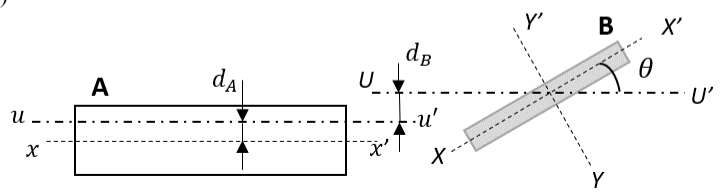

Fig. 2. (a) Cross section of a flexure hinge with flap B in reference state and folded state (gray) (b) Geometry of the skeleton A and flap B with different axes of reference and neutral axes.

We consider a flexure hinge with cross-section shown in Fig. 2(a) and assume that the rectangular regions A forming the skeleton and B forming the flap (initially in the horizontal position) are connected by a rotary joint. For simplicity, we consider the centroids of both regions initially lying in the same horizontal plane shown by the neutral axis $x x^{\prime}$ in Fig. 2(a) and that the regions $\mathrm{A}$ and $\mathrm{B}$ are connected by a simple rotary joint instead of a continuum bending occurring in flap $\mathrm{B}$, as will be seen later in the experimental results. However, the theoretical modelling presented here can be modified to include more complex cross sections. When the flap B rotates about the rotary joint connecting $\mathrm{A}$ and $\mathrm{B}$, attaining the position shown in gray in Fig. 2(a), there is a shift in the neutral axis of the combined structure as well as a change in the second moment of area of the cross-section (Fig. 2(b)). This change in the second moment of area with change in the flap angle is modelled as follows.

The length and width of the rectangular region $\mathrm{A}$ are denoted by $L_{A}$ and $w_{A}$, and that of the rectangular flap $\mathrm{B}$ are denoted by $L_{B}$ and $w_{B}$ respectively. The second moment of area of the rectangle $\mathrm{A}$ about the axis $x x^{\prime}$ is given by:

$$
I_{x x^{\prime}}^{A}=\frac{L_{A} w_{A}^{3}}{12}
$$

The second moment of area of the rectangular flap B about the axes $X X^{\prime}$ and $Y Y^{\prime}$ passing through the centroid are respectively given by:

$$
I_{X X^{\prime}}^{B}=\frac{L_{B} w_{B}^{3}}{12}, I_{Y Y^{\prime}}^{B}=\frac{L_{B}^{3} w_{B}}{12}
$$

Due to the symmetry of rectangle B about axes $X X^{\prime}$ and $Y Y^{\prime}, I_{X Y}=0$. The second moment of inertia of the rectangle B about the axis $U U^{\prime}$ which is obtained by rotating axes $X X^{\prime}$ by an angle $\theta$ is given by:

$$
I_{U U^{\prime}}^{B}=I_{X X^{\prime}}^{B} \cos ^{2} \theta+I_{Y Y^{\prime}}^{B} \sin ^{2} \theta
$$

Due to the thin form factor of the flaps, $L_{B}$ is much greater than $w_{B}$. Thereby, $I_{Y Y^{\prime}}^{B}$ is much greater than $I_{X X^{\prime}}^{B}$. We can note from Eq. (3) that, as $\theta$, the rotation angle of the flap, changes from $0^{o}$ to $90^{\circ}, I_{U U^{\prime}}^{B}$ increases from $I_{X X^{\prime}}^{B}$ to $I_{Y Y^{\prime}}^{B}$. 
Furthermore, the rotation of the flaps also causes the neutral axis to shift to the centroid of the new crosssection. The perpendicular distance of the axis UU' from the combined new centroid of rectangles $A$ and $B$ can be obtained from the distance weighted average of the areas and is given by:

$$
d_{A}=\frac{L_{B} w_{B} \times\left(L_{B} \sin \theta\right) / 2}{L_{A} w_{A}+L_{B} w_{B}}
$$

Similarly, the perpendicular distance from the axis $x x^{\prime}$ to the horizontal axis $u u^{\prime}$ passing through the combined centroid of the rectangles $\mathrm{A}$ and $\mathrm{B}$ is given by:

$$
d_{B}=\frac{L_{A} w_{A} \times\left(L_{B} \sin \theta\right) / 2}{L_{A} w_{A}+L_{B} w_{B}}
$$

The total second moment of area of the rectangles A and B denoted by $I$ is given by applying the parallel axes theorem to translate the individual moments of areas into the new axis of reference $u u^{\prime}$ and summing them up.

$$
I=I_{u u^{\prime}}^{A}+I_{u u^{\prime}}^{B}=\left(I_{X X^{\prime}}^{A}+L_{A} w_{A} \times d_{A}^{2}\right)+\left(I_{U U^{\prime}}^{B}+L_{B} w_{B} \times d_{B}^{2}\right)
$$

Both Eqs. (3) and (6) reveal that the change in the second moment of inertia is mainly a resultant of two factors: 1) the rotation of the flap causing a redistribution of the area of the cross-section in the plane orthogonal to bending and 2) a shift in the neutral axis of the structure. In a culmination of these two factors, change in the angle of the flaps gives rise to increase in the second moment of inertia and consequently, the flexural stiffness of the flexible hinge. This mechanism led to the new design of shape-morphable flexure hinge which is investigated through experiments later in Section IV.

\section{DeSign AND FABRiCATION OF The FleXible FINGER}

\section{A. The 3D printable flexible finger skeleton}

The flexible finger is a single monolithic beam that performs as a compliant kinematic chain jointed by two flexure hinges (Fig.3). The design of the finger skeleton incorporates two separate flexure hinges (Fig.3(b)) of $1 \mathrm{~mm}$ thickness and three stiff links of $3 \mathrm{~mm}$ thickness. These stiff links have a central rectangular cavity of $1 \mathrm{~mm}$ thickness to allow the tendon of the actuation system to pass along the length of the finger. The thicker cross-section of these links makes their flexural rigidity much higher than that of the flexure hinges causing any applied external moments to concentrate the deformation at the flexure hinges.

Along with the geometrical structure that allows for bending in two flexure hinges, the design also incorporates thin and flexible flaps that can be folded using pneumatic pouch actuators offering us the ability to control the stiffness of the flexure hinges. Bordering on either side of a flexural beam, we incorporate a flap of width, $10 \mathrm{~mm}$ and thickness, $0.5 \mathrm{~mm}$. The pneumatic actuators will be adhered to these flaps to rotate them, as will be described later. The proximal
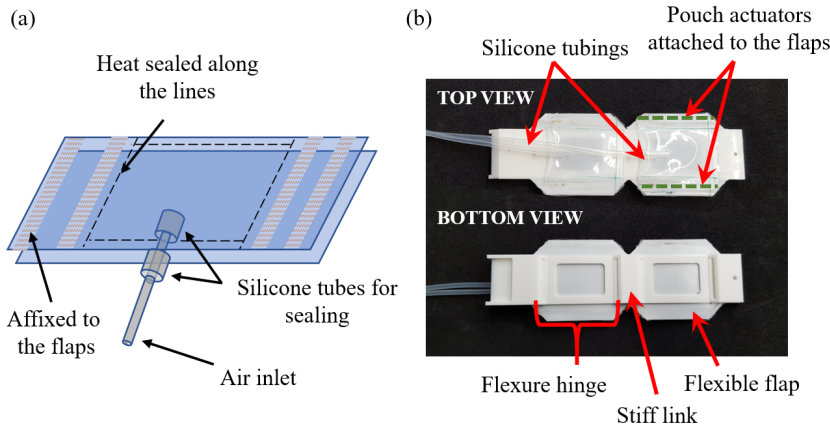

Fig. 3. (a) Fabrication of a pouch actuator (b) Assembly of pouch actuators with the $3 \mathrm{D}$ printed TPU finger

end of the gripper has a slanted geometry with an angle of 26.56 degrees that is used to attach to the motion transmission box in the desired orientation.

Based on the above modelling, the finger skeleton is designed to adopt a multimaterial additive manufacturing process utilising a sacrificial material. The finger structure is $3 \mathrm{D}$ printed from thermoplastic polyurethane (TPU) which is a tough but flexible material and allows elastic strains up to $50 \%$. The fabrication of the bi-layered structure with a thin width and large area is made possible by 3D printing supports made of polyvinyl alcohol (PVA). PVA is a thermoplastic material belonging to the ester family and can dissolve in water. The 3D model incorporating the TPU parts with PVA supports is fabricated using a commercial 3D printer, Ultimaker 3. The printed finger is immersed in water for several hours to let the PVA supports dissolve and any residual PVA material is removed manually using tools.

\section{B. Fabrication of pouch actuators}

Each flexure hinge embeds a pneumatically actuated pouch between the two flaps. Each of the pouches is fabricated from thermoplastic polyurethane (TPU) sheet of thickness $150 \mu \mathrm{m}$. A TPU sheet of dimensions $50 \mathrm{~mm}$ (horizontal) by $100 \mathrm{~mm}$ (vertical) is taken and a tiny hole of $2 \mathrm{~mm}$ diameter is made at the geometric centre of the sheet. The sheet is then folded midway along the vertical edge. This folded sheet is sealed using a heat sealer along two vertical lines, each of them $10 \mathrm{~mm}$ from the edges leaving a gap of $30 \mathrm{~mm}$ between each other (Fig.3). This results in a pouch-like structure with a $2 \mathrm{~mm}$ hole at the bottom and open edge in the top.

A silicone tubing of outer diameter $3 \mathrm{~mm}$ is inserted through the hole at the bottom. Another $10 \mathrm{~mm}$ long silicone tubing with $3 \mathrm{~mm}$ inner diameter and $5 \mathrm{~mm}$ outer diameter is inserted into the open pouch from the open end and the end of the thinner silicone tube lying inside the tube is firmly affixed into the tubing of the larger diameter. The thinner tube is pulled from the outside so that the thicker tubing firmly covers the hole acting as an elastic sealing mechanism. Another silicone tubing of the larger dimensions is also inserted on top of the other end of the tube from outside to firmly seal both sides of the hole. The open end of the pouch is sealed along its boundary using a heat sealer. This 


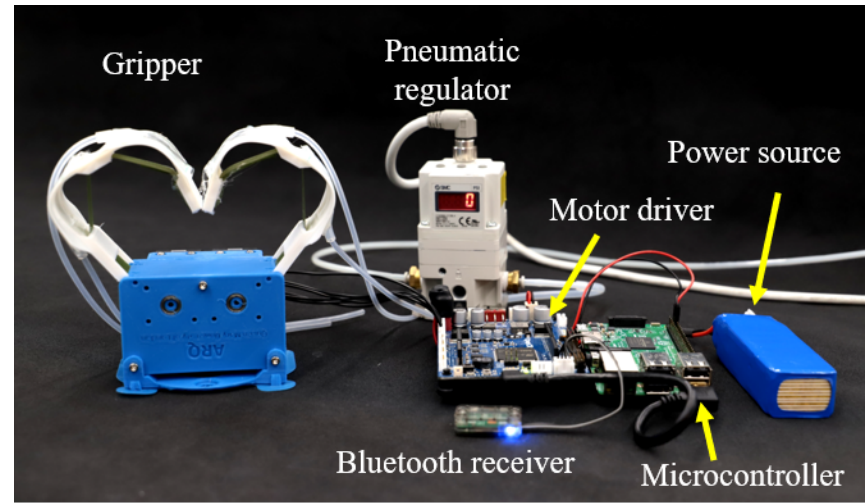

Fig. 4. Prototype and control system of the two-fingered robotic gripper with $3 \mathrm{D}$ printed flexible fingers employing variable stiffness flexure hinges.

results in a fully enclosed pouch with two extruding portions of $10 \mathrm{~mm}$ width on each side. These extruding portions are then affixed to the flaps of flexible TPU finger also through heat sealing technique. Since both the 3D printed flexible finger and the pouches are made from TPU, a firm bond is formed between these two components upon heating and application of pressure.

The silicone tubing is connected to the outlet of a pneumatic pressure regulator, SMC ITV-212BL4. The output pressure of the pressure regulator is controlled by a voltage signal programmed through NI-DAQ 6003 using NILabView $^{T M}$.

\section{Assembly of the two-fingered gripper}

Apart from the TPU skeleton and pouch actuators, the gripper has two more functional components, which are motors and tendons. Two Dynamixel XM430-210-R smart servo motors are lodged inside a $3 \mathrm{D}$ printed motor transmission box fitted with flanged bearings. A fabric tendon made of TPU coated nylon is affixed to the shaft of each servo motor. The fabric tendon is then run along the length of the 3D printed flexible finger through the grooves in the TPU skeleton and attached to the distal end of the TPU finger such that the fabric tendon is in tension when the finger is fully flat.

The two Dynamixel servos are controlled using a Raspberry Pi 3 Model B microcontroller installed with ROS Kinetic. An Open CR1.0 motor driver controlled using Arduino IDE is used to reconfigure and drive the servo motors. The motor drivers are calibrated upon connection, using $\mathrm{R}+$ manager. Upon configuration of the motors, a Bluetooth master slave RC-100 controller is used to control the speed of the motors with a handheld remote control.

\section{Stiffness Control of the Flexure Hinges}

\section{A. Stiffness of flexure hinges with different flap angles}

Based on the modelling in Section II, we understand the morphing of flaps of the TPU fingers causes a variation in their flexural stiffness. In order to test this concept experimentally, we 3D print three TPU fingers with varying flaps folding angles (denoted by $\theta_{\text {flap }}$ ) of $15^{\circ}, 45^{\circ}$ and $60^{\circ}$ respectively. A mounting structure is $3 \mathrm{D}$ printed with a rigid material, polylactic acid (PLA). A flexure hinge is affixed to this mounting structure using nuts and bolts, which is affixed to the base of an Instron 3342 Universal Testing Machine installed with a $0.5 \mathrm{kN}$ load cell with an accuracy range of $\pm 1 \%$ at $1 \mathrm{~N}$. A line indenter is mounted on the load cell of the Universal Testing Machine. The indenter is programmed to indent the tip of the TPU finger at a slow rate of $10 \mathrm{~mm} / \mathrm{min}$ to simulate a quasi-static loading condition. The load-deflection curves for the three different configurations of the flexure hinge are shown in Fig. 5(a). We note that as the folding angle of the flaps increases, the load required to cause the same deflection increases, indicating an increase in the flexural stiffness. With these results indicating that the flap angle influences the flexural rigidity of the flexure hinge, we proceed to integrate pneumatic pouch actuators to achieve shape morphing of the flexure hinge.
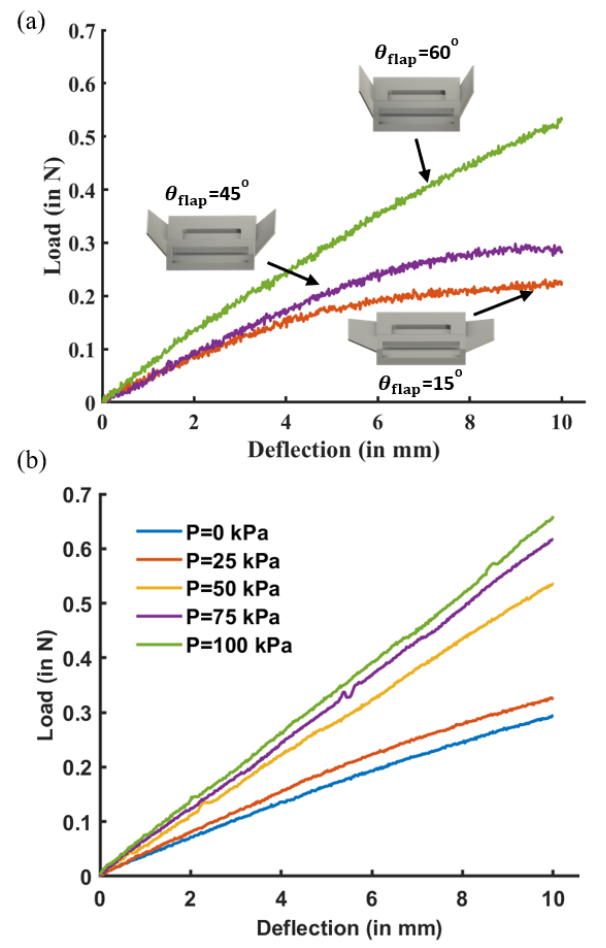

Fig. 5. Effect of shape morphing on the flexural stiffness of a flexure hinge, Force vs lateral displacement for (a) prefabricated flexure hinges with flap angles of 15, 45 and 60 degrees, and (b) a flexure hinge embedded with pneumatic pouch actuator at different actuation pressures

\section{B. Pneumatically actuated shape-morphing of the flexure hinge}

A pneumatic pouch actuator is integrated between the two foldable flaps of a 3D printed flexure hinge. When the pouch is actuated with pressure, it is inflated from a flat to spherical shape without experiencing significant expansion of the sheet material. The change in geometry of the pouches from flat to spherical results in an overall linear contraction between the two ends pulling the flaps towards the centre causing them 


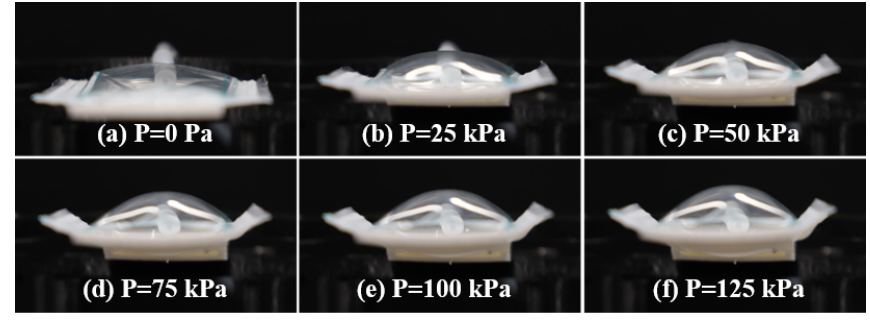

Fig. 6. Images showing deformation of the shell structure at different pouch pressures

to fold. In this process, the contraction forces of the pouches exert the moments required to fold the flaps and are both in equilibrium. Hence, the deformation of the flaps is dependent on the actuation pressure of the pouches at the given instant. This deformation is examined by actuating the pouches to different set pressures and the cross sectional views of the flexure hinge with the flaps are captured using a camera (Fig. $6)$.

When no pressure is applied, the flaps are in a flat configuration. As the pressure is increased to $25 \mathrm{kPa}$, the pouches inflate slightly causing a deflection of around 19 degrees. Increasing pressure to $50 \mathrm{kPa}$, it results in a significant contraction of the pouches and the flaps bend to reach 34 degrees deflection at the tip. When the pressure is increased further to $75 \mathrm{kPa}$ and $100 \mathrm{kPa}$ respectively, the deflection of the flaps increases only marginally to 38 and 42 degrees respectively. This behaviour can be explained from the fact that the pouch actuators have a limiting actuation strain which is reached when the volume of the pouch is maximised. The maximum theoretical strain achievable in a pouch actuator is $36 \%$ which corresponds to the case when the cross section of the pouch forms a circle. This actuation strain can be realised in an ideal scenario when the pouch actuator is made of very thin sheets with negligible bending rigidity, has a slender form factor with its width much greater than the length and is unobstructed in the out of plane directions. However, in the pouch actuator applied to the flaps, the pouch has a length and width of $30 \mathrm{~mm}$ and $4 \mathrm{~mm}$ respectively and the deformation of the pouch is obstructed on one side by the TPU skeleton resulting in a lower actuation strain.

\section{Stiffness of flexure hinge with pouch actuated flaps}

Towards the application of stiffness controlled hinges embedded with pouch actuators, here we investigate the effect of actuation pressure on the flexural stiffness of a flexure hinge embedded with a pouch actuator. A flexure hinge is mounted onto a mechanical test rig on an Instron 3342 Universal Testing Machine installed with a $0.5 \mathrm{kN}$ load cell. Different actuation pressures are applied to the pouch and for each pouch pressure, the finger is indented at a rate of $10 \mathrm{~mm} / \mathrm{min}$. The load-deflection curves at different pouch pressures are shown in Fig. 5(b). As expected, we see that as the pressure in the pouches increases, the flexural stiffness of the finger increases. Furthermore, we note that the largest change in the stiffness occurs when the pouch pressure is

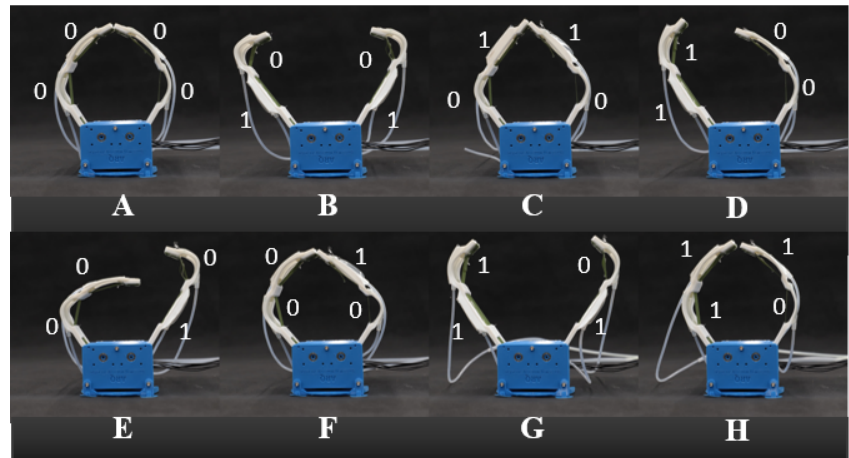

Fig. 7. Actuation of different pouches leads to different gripper configurations upon actuation by tendons. 1 and 0 represent whether the corresponding pouch at the flexure hinge is actuated or unactuated respectively.

changed from $25 \mathrm{kPa}$ to $50 \mathrm{kPa}$. This corresponds to the large change in deflection of the flaps as noted in subsection IV-A. As the deflection of the flaps saturates at higher pressures, the marginal increase in the stiffness is also low.

\section{GRASPING USING Two-FINGERED GRIPPER WITH Stiffness Controllable FleXURe Hinges}

The ability of the pneumatic pouches to control the flexural stiffness of the flexible fingers offers the unique potential to achieve different configurations by tendon actuation resulting in different grasp modes. In this section, we test the various combinations in which the pouches embedded in the two flexible fingers can be actuated and realise the different configurations achievable by the underactuated gripper. There are a total of eight unique configurations in which the four pouches can be actuated eliminating configuration which are symmetric (Fig. 7). When the number close to a flexure hinge reads 1 , it means the corresponding pouch is actuated to $50 \mathrm{kPa}$ in that particular scenario. A pneumatic pressure of $50 \mathrm{kPa}$ is utilized since the experimental results in Fig.5(b) show that it is sufficient to cause a significant increase in the flexural stiffness of the flexure hinge.

In scenario $\mathrm{A}$, we observe that the bottom hinges flex when the servos are actuated. This is because the tendons are attached to the distal end of the flexible finger and the moment about the bottom flexure hinge is higher than that about the top flexure hinge due its greater distance from the point of force applied. Hence, in the default mode in which none of the pouches are pneumatically actuated, the bottom flexure hinge deforms first. In scenario B, the bottom pouches in both the left and right fingers are actuated. This causes the bottom flexure hinges to stiffen resulting in significant deformation in the top hinges when the servos are actuated. Combinations of different actuation sequences and the resulting configurations of the grippers are shown in Fig. 7.

With the wide range of configurations that can be achieved by the gripper and the high conformability of the flexible fingers, we demonstrate the versatility of the gripper in grasping different objects encountered in daily life (Fig. 8(a)). It can be seen that the gripper can handle objects with 


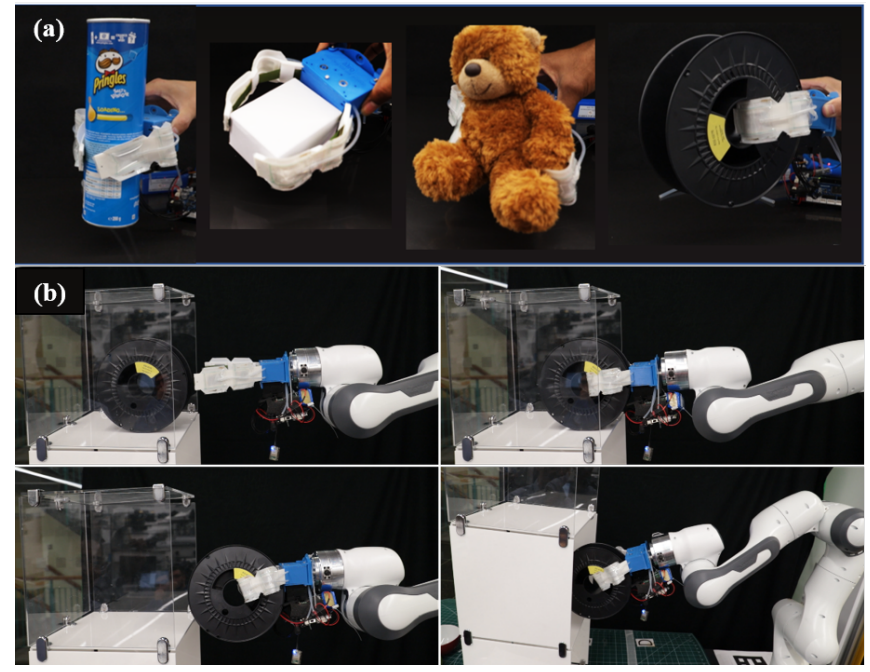

Fig. 8. (a) Two-fingered gripper grasping different everyday objects (b) Image sequence showing the two-fingered gripper mounted on a Franka robot arm grasping an object from the top shelf and transferring it to the bottom shelf

different shapes and sizes and with stiffness ranging from rigid to soft. Further, the high compliance of the gripper makes it well-suited for deployment in unstructured and congested environments where interaction with the environment is likely. To demonstrate this capability, we mounted the developed gripper onto the end-effector of a Franka robot arm and performed a pick and place operation through a teleoperation protocol (Fig. 8(b)). The gripper is first manoeuvred to reach into a confined shelf with a spool. On reaching the spool, the servos are actuated through a remote control and flexible fingers firmly grasp the spool. The robot arm is then teleoperated to take the spool out of the shelf and place it into another shelf underneath. Once the spool is completely inside the bottom shelf, the gripper is released to unload the spool. This demonstration showcases the unique advantages offered by the gripper for tasks in confined space. What is more, the wide range of configurations offered by the control of stiffness of the flexure hinges can be leveraged to undertake fine operations in such confined spaces.

\section{CONCLUSIONS}

This paper presented a novel approach for developing soft robotic grippers with variable stiffness hinges in terms of the theory of plates and beams. Using the proposed concept, a flexure hinge composed of a compliant beam and two morphable flaps was deigned and fabricated using multi-material $3 \mathrm{D}$ printing. A pneumatic pouch actuator was produced and integrated with the new flexure hinge to fold and unfold its flaps from plate to open section beam structure, leading to stiffness variation of the flexure hinge. The morphing of the flaps and the stiffness variation of single flexure hinge under various air pressures in the pouch actuator was evaluated with static tests. The experimental results verified the analytical model and design concept of stiffness control by changing the cross section of the flexure hinge. Utilizing the variable stiffness flexure hinges as joints of flexible robotic fingers, a prototype of a two-fingered tendon driven robotic gripper was developed and a control scheme for realizing various grasp modes of the gripper were demonstrated using the prototype. The experimental grasps of both rigid and soft material objects with various shape demonstrated that the new gripper can be used in picking and placing operation in storage and confined environments.

\section{ACKNOWLEDGMENT}

We thank Mr. Bukeikhan Omarali for his help with the teleoperation setup using the Franka robot arm.

\section{REFERENCES}

[1] D. Rus and M. T. Tolley, "Design, fabrication and control of soft robots," Nature, vol. 521, no. 7553, p. 467, 2015.

[2] C. Majidi, "Soft robotics: a perspective-current trends and prospects for the future," Soft Robotics, vol. 1, no. 1, pp. 5-11, 2014.

[3] L. Birglen, T. Laliberté, and C. M. Gosselin, Underactuated robotic hands. Springer, 2007, vol. 40.

[4] C. M. Gosselin and T. Laliberte, "Underactuated mechanical finger with return actuation," Jun. 9 1998, uS Patent 5,762,390.

[5] T. Laliberté, L. Birglen, and C. Gosselin, "Underactuation in robotic grasping hands," Machine Intelligence \& Robotic Control, vol. 4, no. 3, pp. 1-11, 2002.

[6] M. Baril, T. Laliberté, C. Gosselin, and F. Routhier, "On the design of a mechanically programmable underactuated anthropomorphic prosthetic gripper," Journal of Mechanical Design, vol. 135, no. 12, p. $121008,2013$.

[7] B. Massa, S. Roccella, M. C. Carrozza, and P. Dario, "Design and development of an underactuated prosthetic hand," Proceedings 2002 IEEE International Conference on Robotics and Automation (Cat. No.02CH37292), vol. 4, pp. 3374-3379 vol.4, 2002.

[8] Y. Tlegenov, K. Telegenov, and A. Shintemirov, "An open-source $3 \mathrm{~d}$ printed underactuated robotic gripper," in 2014 IEEE/ASME 10th International Conference on Mechatronic and Embedded Systems and Applications (MESA). IEEE, 2014, pp. 1-6.

[9] J. Shintake, V. Cacucciolo, D. Floreano, and H. Shea, "Soft robotic grippers," Advanced Materials, vol. 30, no. 29, p. 1707035, 2018.

[10] M. Bonilla, E. Farnioli, C. Piazza, M. Catalano, G. Grioli, M. Garabini, M. Gabiccini, and A. Bicchi, "Grasping with soft hands," in 2014 IEEE-RAS International Conference on Humanoid Robots. IEEE, 2014, pp. 581-587.

[11] L. U. Odhner, L. P. Jentoft, M. R. Claffee, N. Corson, Y. Tenzer, R. R. Ma, M. Buehler, R. Kohout, R. D. Howe, and A. M. Dollar, "A compliant, underactuated hand for robust manipulation," The International Journal of Robotics Research, vol. 33, no. 5, pp. 736-752, 2014.

[12] M. Manti, T. Hassan, G. Passetti, N. D'Elia, C. Laschi, and M. Cianchetti, "A bioinspired soft robotic gripper for adaptable and effective grasping," Soft Robotics, vol. 2, no. 3, pp. 107-116, 2015.

[13] R. R. Ma, A. Spiers, and A. M. Dollar, "M 2 gripper: Extending the dexterity of a simple, underactuated gripper," in Advances in reconfigurable mechanisms and robots II. Springer, 2016, pp. 795805.

[14] J. Hughes, P. Maiolino, and F. Iida, "An anthropomorphic soft skeleton hand exploiting conditional models for piano playing," Science Robotics, vol. 3, no. 25, p. eaau3098, 2018.

[15] K. C. Galloway, K. P. Becker, B. Phillips, J. Kirby, S. Licht, D. Tchernov, R. J. Wood, and D. F. Gruber, "Soft robotic grippers for biological sampling on deep reefs," Soft robotics, vol. 3, no. 1, pp. 23-33, 2016

[16] J. Fras and K. Althoefer, "Soft biomimetic prosthetic hand: Design, manufacturing and preliminary examination," in 2018 IEEE/RSJ International Conference on Intelligent Robots and Systems (IROS), Oct 2018, pp. 1-6.

[17] K. Ham, J. Han, and Y.-J. Park, "Soft gripper using variable stiffness mechanism and its application," International Journal of Precision Engineering and Manufacturing, vol. 19, no. 4, pp. 487-494, 2018.

[18] A. Firouzeh and J. Paik, "An under-actuated origami gripper with adjustable stiffness joints for multiple grasp modes," Smart Materials and Structures, vol. 26, no. 5, p. 055035, 2017. 